\title{
Hom complexes of set systems
}

\author{
Jakob Jonsson* \\ Department of Mathematics \\ KTH Royal Institute of Technology \\ Stockholm, Sweden \\ jakobj@kth.se
}

Submitted: Feb 20, 2012; Accepted: Dec 24, 2012; Published: Jan 7, 2013

Mathematics Subject Classifications: 05E45, 55U10, 55S35

\begin{abstract}
A set system is a pair $\mathcal{S}=(V(\mathcal{S}), \Delta(\mathcal{S}))$, where $\Delta(\mathcal{S})$ is a family of subsets of the set $V(\mathcal{S})$. We refer to the members of $\Delta(\mathcal{S})$ as the stable sets of $\mathcal{S}$. A homomorphism between two set systems $\mathcal{S}$ and $\mathcal{T}$ is a map $f: V(\mathcal{S}) \rightarrow V(\mathcal{T})$ such that the preimage under $f$ of every stable set of $\mathcal{T}$ is a stable set of $\mathcal{S}$. Inspired by a recent generalization due to Engström of Lovász's Hom complex construction, the author associates a cell complex $\operatorname{Hom}(\mathcal{S}, \mathcal{T})$ to any two finite set systems $\mathcal{S}$ and $\mathcal{T}$. The main goal of the paper is to examine basic topological and homological properties of this cell complex for various pairs of set systems.
\end{abstract}

Keywords: Hom complex, set system, partitionable poset

\section{Introduction}

Recall that a graph homomorphism from a graph $G=(V, E)$ to a graph $G^{\prime}=\left(V^{\prime}, E^{\prime}\right)$ is a map $f: V \rightarrow V^{\prime}$ such that $f(x)$ and $f(y)$ are adjacent in $G^{\prime}$ whenever $x$ and $y$ are adjacent in $G$. Lovász showed how to define a cell complex $\operatorname{Hom}\left(G, G^{\prime}\right)$ with the property that the vertices are indexed by the graph homomorphisms from $G$ to $G^{\prime}$; see Babson and Kozlov [1, 2] and Kozlov [12].

A vertex set in a graph $G$ is independent if no two vertices in the set are joined by an edge. The independence complex $\operatorname{Ind}(G)$ is the simplicial complex of independent sets in $G$. Note that a map $f: V \rightarrow V^{\prime}$ is a graph homomorphism if and only if $f^{-1}(\sigma) \in \operatorname{Ind}(G)$ for each $\sigma \in \operatorname{Ind}\left(G^{\prime}\right)$. Inspired by this observation, Engström [6] generalized the construction to arbitrary pairs of simplicial complexes. More precisely, given any abstract simplicial complexes $\Delta$ and $\Delta^{\prime}$ on the vertex sets $V$ and $V^{\prime}$, respectively, say that a map $f: V \rightarrow V^{\prime}$

*Supported by the Swedish Research Council (grant 2006-3279). 
is a homomorphism from $\Delta$ to $\Delta^{\prime}$ if $f^{-1}(\sigma) \in \Delta$ for each $\sigma \in \Delta^{\prime}$. This gives rise to a cell complex in which the vertices are indexed by the homomorphisms from $\Delta$ to $\Delta^{\prime}$. Engström's construction has interesting connections to Ramsey theory.

In this paper, we take Engström's generalization one step further, dropping the requirement that $\Delta$ and $\Delta^{\prime}$ are simplicial complexes. Instead, they can be arbitrary families of subsets of given ground sets $V$ and $V^{\prime}$, respectively. We refer to the pairs $\mathcal{S}=(V, \Delta)$ and $\mathcal{S}^{\prime}=\left(V^{\prime}, \Delta^{\prime}\right)$ as set systems. The members of $\Delta$ and $\Delta^{\prime}$ are the stable sets of $\mathcal{S}$ and $\mathcal{S}^{\prime}$, respectively. A straightforward adaptation of Lovász's construction yields a cell complex $\operatorname{Hom}\left(\mathcal{S}, \mathcal{S}^{\prime}\right)$ with one vertex for each homomorphism from $\mathcal{S}$ to $\mathcal{S}^{\prime}$. We discuss set systems in Section 2 and introduce the associated cell complexes in Section 5.

The original motivation for the generalization was to introduce new tools for examining interval partitions of partially ordered sets (posets). For a given poset $P=(V$, $\leqslant)$, let $I(P)$ denote the family of closed intervals in $P$; see Section 2 for details. By convention, the empty set is an interval. We refer to the set system $\mathcal{I}(P)=(V, I(P))$ as the interval system of $P$. For $n \geqslant 1$, there is a bijective correspondence between ordered partitions of $P$ into $n$ closed intervals and homomorphisms from the interval system of $P$ to the discrete system

$$
\mathcal{D}_{n}=(\{1, \ldots, n\},\{\emptyset,\{1\}, \ldots,\{n\}\}) .
$$

Specifically, a given partition $\left(\sigma_{1}, \ldots, \sigma_{n}\right)$ corresponds to the homomorphism $f: \mathcal{I}(P) \rightarrow$ $\mathcal{D}_{n}$ defined by $f^{-1}(\{k\})=\sigma_{k}$ for $k \in\{1, \ldots, n\}$.

For an important special case, let $\Lambda$ be a finite simplicial complex. We obtain a poset structure on $\Lambda$ by ordering its faces by inclusion. We say that $\Lambda$ is partitionable if $\Lambda$ admits a partition into closed intervals such that each interval contains a maximal face. Stanley [18, Conjecture 2.7] conjectured that $\Lambda$ is partitionable whenever $\Lambda$ is a CohenMacaulay complex. We generalize the concept of partitionability to arbitrary set systems, saying that a set system $\mathcal{S}$ is partitionable if there is an integer $n$ such that there are homomorphisms both from $\mathcal{D}_{n}$ to $\mathcal{S}$ and from $\mathcal{S}$ back to $\mathcal{D}_{n}$, where $\mathcal{D}_{n}$ is the discrete system defined in (1). See Section 3 for details.

Let $\mathcal{X}$ and $\mathcal{S}$ be set systems, and assume that $m$ is maximal such that there is a homomorphism from $\mathcal{D}_{m}$ to $\mathcal{S}$. Any homomorphism from $\mathcal{D}_{m}$ to $\mathcal{S}$ gives rise to a cellular map from $\operatorname{Hom}\left(\mathcal{X}, \mathcal{D}_{m}\right)$ to $\operatorname{Hom}(\mathcal{X}, \mathcal{S})$. In Section 5, we show that the induced map in reduced homology is a monomorphism whenever $\mathcal{S}$ is partitionable. This yields a necessary topological condition for a set system to be partitionable. In particular, the result provides a tool for detecting non-partitionability: if the induced homology map is not a monomorphism, then $\mathcal{S}$ is not partitionable. We say that $\mathcal{X}$ is a witness for the non-partitionability of $\mathcal{S}$.

The result has limitations. For the special case that $\mathcal{X}$ is monotone (the stable sets of $\mathcal{X}$ form a simplicial complex) and $\mathcal{S}$ is the interval system of a simplicial complex, it turns out that the above homology map is always a monomorphism. As a consequence, we cannot use monotone systems as witnesses in this case. See Section 6.2 for more information.

In Section 7, we give examples showing that there exist non-monotone set systems that are, indeed, witnesses for the non-partitionability of some simplicial complexes. The 
complexes under consideration are easily seen to be non-partitionable by direct examination; hence the examples do not shed much light on partitionability of complexes, let alone Stanley's conjecture. What is still good news is the very existence of witnesses, given the absence of witnesses among monotone systems.

A particularly nice example is the interval system $\mathcal{I}\left(L_{3}\right)$ of the 3 -chain $L_{3}$. This system benefits from having a quite simple structure, allowing for a detailed analysis of the associated Hom complexes. To avoid obscuring the paper with too many technical details, we confine ourselves to proving the following two results; see Section 7.1 for details and further directions.

1. The complex $\operatorname{Hom}\left(\mathcal{I}\left(L_{3}\right), \mathcal{D}_{n}\right)$ is homotopy equivalent to a wedge of $n-1$ spheres of dimension $n-1$.

2. The system $\mathcal{I}\left(L_{3}\right)$ is a witness for the non-partitionability of any simplicial complex containing a link isomorphic to the disjoint union of two edges.

In Section 8, we round up the paper with a discussion on the categorical product and coproduct of set systems. The coproduct turns out to be similar to the join operation on simplicial complexes. The exact shape of the product depends on whether we consider the full category of all set systems or the subcategory of monotone systems.

\section{Set systems and homomorphisms}

We define a set system to be a pair $\mathcal{S}=(V(\mathcal{S}), \Delta(\mathcal{S})$ ), where $\Delta(\mathcal{S})$ is a family of subsets of the nonempty set $V(\mathcal{S})$. We refer to $V(\mathcal{S})$ as the ground set and to the members of $\Delta(\mathcal{S})$ as the stable sets of $\mathcal{S}$. Following Engström's approach [6], define a homomorphism between two set systems $\mathcal{S}$ and $\mathcal{T}$ to be a map $f: V(\mathcal{S}) \rightarrow V(\mathcal{T})$ such that $f^{-1}(\tau) \in \Delta(\mathcal{S})$ for every $\tau \in \Delta(\mathcal{T})$. In words, the preimage under $f$ of every stable set of $\mathcal{T}$ is a stable set of $\mathcal{S}$. Let $\operatorname{Hom}_{0}(\mathcal{S}, \mathcal{T})$ denote the set of homomorphisms from $\mathcal{S}$ to $\mathcal{T}$.

Lemma 1. If $f \in \operatorname{Hom}_{0}(\mathcal{S}, \mathcal{T})$ and $g \in \operatorname{Hom}_{0}(\mathcal{T}, \mathcal{U})$, then $g f \in \operatorname{Hom}_{0}(\mathcal{S}, \mathcal{U})$.

Proof. Let $\sigma$ be a stable set of $\mathcal{U}$. Then $g^{-1}(\sigma)$ is stable in $\mathcal{T}$, because $g$ is a homomorphism, and $f^{-1}\left(g^{-1}(\sigma)\right)=(g f)^{-1}(\sigma)$ is stable in $\mathcal{S}$, because $f$ is a homomorphism.

Let us list some basic set systems. Throughout the list, $\mathcal{S}$ denotes an arbitrary set system.

- To every nonempty set $U$, we may associate the void system $(U, \emptyset)$. Every map $f$ : $V(\mathcal{S}) \rightarrow U$ defines a homomorphism from $\mathcal{S}$ to $(U, \emptyset)$. There are no homomorphisms from $(U, \emptyset)$ to $\mathcal{S}$ unless $\mathcal{S}$ is also void.

- We also have the full system $\left(U, 2^{U}\right)$. Every map $f: U \rightarrow V(\mathcal{S})$ defines a homomorphism from $\left(U, 2^{U}\right)$ to $\mathcal{S}$. 
- Of particular importance is the discrete system $\mathcal{D}_{n}$ defined in (1). A map $f$ : $\{1, \ldots, n\} \rightarrow V(\mathcal{S})$ is a homomorphism from $\mathcal{D}_{n}$ to $\mathcal{S}$ if and only if the preimage of every stable set of $\mathcal{S}$ consists of at most one element. A map $f: V(\mathcal{S}) \rightarrow\{1, \ldots, n\}$ is a homomorphism from $\mathcal{S}$ to $\mathcal{D}_{n}$ if and only if the preimage of every $k \in\{1, \ldots, n\}$ is a stable set of $\mathcal{S}$. If $\mathcal{S}=\mathcal{D}_{m}$, then $f$ defines a homomorphism if and only if $f$ is injective.

We proceed with some interesting classes of set systems and homomorphisms.

- Let $G=(V, E)$ be a graph. The independence system of $G$ is the set system $(V, \operatorname{Ind}(G))$, where $\operatorname{Ind}(G)$ is the independence complex of $G$. As mentioned, a homomorphism between the independence systems of two graphs $G$ and $H$ is the same as a graph homomorphism between $G$ and $H$. Note that the discrete system $\mathcal{D}_{n}$ is the independence system of the complete loopless graph $K_{n}$.

- A set system $\mathcal{S}=(V, \Delta)$ is monotone if $\Delta$ is a simplicial complex. This is equivalent to saying that $\rho \in \Delta$ whenever there exists some $\sigma \in \Delta$ such that $\rho \subset \sigma$. This is the class of set systems that Engström [6] examined.

- Let $P=\left(V(P), \leqslant_{P}\right)$ be a finite poset on the ground set $V(P)$. For any elements $a, b \in V(P)$ such that $a \leqslant_{P} b$, define

$$
[a, b]=\left\{x \in V(P): a \leqslant_{P} x \leqslant_{P} b\right\} .
$$

This is the (closed) interval in $P$ between $a$ and $b$. Define the interval system of $P$ to be the set system $\mathcal{I}(P)=(V(P), I(P))$, where

$$
I(P)=\{\emptyset\} \cup\left\{[a, b]: a \leqslant_{P} b\right\} .
$$

Interval systems are typically not monotone. For example, if $P$ is the chain $x<y<$ $z$, then $I(P)$ contains $\{x, y, z\}$ but not $\{x, z\}$.

- An important variant of the interval system of a poset $P$ is the reduced interval system of $P$, which we denote by $\mathcal{I}^{\text {red }}(P)$. This is the set system on $V(P)$ in which the stable sets are the empty set and all closed intervals of $P$ that contain a coatom. By a coatom, we mean an element $x \in V(P)$ such that no element $y \in V(P)$ satisfies $x<_{P} y$.

\section{$3 \quad$ Partitions and copartitions}

An interval partition of a poset $P$ is an ordered partition $\left(I_{1}, \ldots, I_{n}\right)$ of $V(P)$ such that each $I_{j}$ is a closed interval or empty. Equivalently, each $I_{j}$ is stable in the interval system $\mathcal{I}(P)$. In particular, the existence of an interval partition of size $n$ is equivalent to the existence of a homomorphism $f \in \operatorname{Hom}_{0}\left(\mathcal{I}(P), \mathcal{D}_{n}\right)$. Namely, such a homomorphism $f$ induces the interval partition $\left(f^{-1}(1), \ldots, f^{-1}(n)\right)$. 
More generally, we define an $n$-partition of a set system $\mathcal{S}$ to be a homomorphism $f \in \operatorname{Hom}_{0}\left(\mathcal{S}, \mathcal{D}_{n}\right)$. Define $\chi(\mathcal{S})$ to be the smallest $n \geqslant 1$ such that $\mathcal{S}$ admits an $n$ partition. By convention, $\chi(\mathcal{S})=\infty$ if there is no $n$-partition for any $n$.

Dually, an $m$-copartition of $\mathcal{S}$ is a homomorphism $f \in \operatorname{Hom}_{0}\left(\mathcal{D}_{m}, \mathcal{S}\right)$. Equivalently, an $m$-copartition $f$ has the property that $f^{-1}(\sigma)$ consists of at most one element for each stable set $\sigma$. We identify an $m$-copartition $f$ with the sequence $(f(1), \ldots, f(m))$. Define $\omega(\mathcal{S})$ to be the greatest $m \geqslant 1$ such that $\mathcal{S}$ admits an $m$-copartition. Note that $\omega(\mathcal{S}) \geqslant 1$ for any $\mathcal{S}$, because $\mathcal{D}_{1}$ is a full system.

\subsection{Basic properties of partitions and copartitions}

We state and prove some very basic facts about the numbers $\chi(\mathcal{S})$ and $\omega(\mathcal{S})$ for a given set system $\mathcal{S}$.

Proposition 2. For any set system $\mathcal{S}$, we have that $\omega(\mathcal{S}) \leqslant \chi(\mathcal{S})$.

Proof. Suppose that $f \in \operatorname{Hom}_{0}\left(\mathcal{D}_{m}, \mathcal{S}\right)$ and $g \in \operatorname{Hom}_{0}\left(\mathcal{S}, \mathcal{D}_{n}\right)$. Then we have that $g f \in$ $\operatorname{Hom}_{0}\left(\mathcal{D}_{m}, \mathcal{D}_{n}\right)$ by Lemma 1 . Since every homomorphism from $\mathcal{D}_{m}$ to $\mathcal{D}_{n}$ is injective, we deduce that $m \leqslant n$.

Proposition 3. Let $\mathcal{S}$ and $\mathcal{T}$ be set systems. If $\operatorname{Hom}_{0}(\mathcal{S}, \mathcal{T})$ is nonempty, then $\omega(\mathcal{S}) \leqslant$ $\omega(\mathcal{T})$ and $\chi(\mathcal{S}) \leqslant \chi(\mathcal{T})$.

Proof. Let $g \in \operatorname{Hom}_{0}(\mathcal{S}, \mathcal{T})$. If $h \in \operatorname{Hom}_{0}\left(\mathcal{T}, \mathcal{D}_{n}\right)$, then Lemma 1 implies that $h g \in$ $\operatorname{Hom}_{0}\left(\mathcal{S}, \mathcal{D}_{n}\right)$; hence $\mathcal{S}$ admits an $n$-partition whenever $\mathcal{T}$ does. Analogously, if $f \in$ $\operatorname{Hom}_{0}\left(\mathcal{D}_{m}, \mathcal{S}\right)$, then $g f \in \operatorname{Hom}_{0}\left(\mathcal{D}_{m}, \mathcal{T}\right)$; hence $\mathcal{T}$ admits an $m$-copartition whenever $\mathcal{S}$ does.

We say that $\mathcal{S}$ is weakly partitionable if $\mathcal{S}$ admits a partition and strongly partitionable if $\mathcal{S}$ admits a partition of size $\omega(\mathcal{S})$. For simplicity, we refer to strongly partitionable set systems simply as partitionable. To summarize, $\mathcal{S}$ is partitionable if and only if $\omega(\mathcal{S})=\chi(\mathcal{S})$

For example, suppose that $\mathcal{S}$ is the independence system of a given graph $G$. Then $\omega(\mathcal{S})$ is the size of a largest clique in $G$, whereas $\chi(\mathcal{S})$ is the chromatic number of $G$. In particular, $\mathcal{S}$ is partitionable if and only if the clique number and the chromatic number of $G$ coincide.

We proceed with a partial characterization of set systems $\mathcal{S}$ such that $\omega(\mathcal{S})$ is finite. Refer to a set system $\mathcal{S}$ as free if each $v \in V(\mathcal{S})$ is contained in some stable set of $\mathcal{S}$. See Section 6.1 for some motivation for this terminology.

Proposition 4. Let $\mathcal{S}$ be a set system. If $\omega(\mathcal{S})<\infty$, then $\mathcal{S}$ is free. The converse is true if $|V(\mathcal{S})|<\infty$.

Proof. Suppose that $\mathcal{S}$ is not free. Let $v \in V(\mathcal{S})$ be such that no stable set of $\mathcal{S}$ contains $v$. Then the constant map $k \mapsto v$ defines a homomorphism from $\mathcal{D}_{m}$ to $\mathcal{S}$ for any choice of $m$. Conversely, suppose that $\mathcal{S}$ is free, and let $f \in \operatorname{Hom}_{0}\left(\mathcal{D}_{m}, \mathcal{S}\right)$. Then $f$ is injective, because each $v \in V(\mathcal{S})$ belongs to a stable set, whose preimage has size at most one. We conclude that $m$ cannot exceed the cardinality of $V(\mathcal{S})$. 
Remark 5. One may generalize the theory by introducing infinite discrete systems, defining $\omega(\mathcal{S})$ to be the smallest cardinal number $c$ such that there is a homomorphism from the discrete system of cardinality $c$ to $\mathcal{S}$, and defining $\chi(\mathcal{S})$ analogously. We leave the details to the interested reader.

\subsection{Partitions and copartitions of interval systems}

As many of our examples will be interval systems of posets as defined in Section 2, we discuss such systems in some detail.

For a given poset $P$, write $\chi(P)=\chi(\mathcal{I}(P))$; this is the minimum value $n$ such that $\operatorname{Hom}_{0}\left(\mathcal{I}(P), \mathcal{D}_{n}\right)$ is nonempty. Moreover, write $\omega(P)=\omega(\mathcal{I}(P))$; this is the maximum value $m$ such that $\operatorname{Hom}_{0}\left(\mathcal{D}_{m}, \mathcal{I}(P)\right)$ is nonempty. By Proposition 2 , we have that $\omega(P) \leqslant$ $\chi(P)$. We say that $P$ is partitionable if $\omega(P)=\chi(P)$. This is equivalent to saying that the interval system $\mathcal{I}(P)$ is (strongly) partitionable.

A poset $P$ is rooted if there exists a unique minimal element in $P$. Such an element 0 has the property that $0 \leqslant P$ for all $x$ in $P$.

Proposition 6. If $P$ is a rooted poset, then $\omega(P)$ equals the number of coatoms of $P$.

Proof. Let $c_{1}, \ldots, c_{m}$ be the coatoms of $P$. We obtain a homomorphism from $\mathcal{D}_{m}$ to $\mathcal{I}(P)$ by defining $f(k)=c_{k}$ for each $k$. To see that this is indeed a homomorphism, note that any interval $\sigma$ in $P$ contains at most one coatom; hence $f^{-1}(\sigma)$ contains at most one element and is therefore stable in $\mathcal{D}_{m}$. Conversely, suppose that we have a homomorphism from $\mathcal{D}_{n}$ to $\mathcal{I}(P)$ for some $n$. Then $f^{-1}\left(\left[0, c_{i}\right]\right)$ contains at most one element for each $i$, where 0 is the minimal element in $P$. Since $V(P)$ is the union of the intervals $\left[0, c_{1}\right], \ldots,\left[0, c_{m}\right]$, we deduce that $f^{-1}(V(P))$ contains at most $m$ elements; hence $n \leqslant m$.

Corollary 7. Let $P$ be a rooted poset with minimal element 0 and $m$ coatoms $c_{1}, \ldots, c_{m}$. Then $P$ is partitionable if and only if there exists a homomorphism $f$ from $\mathcal{I}(P)$ to $\mathcal{D}_{m}$ and elements $b_{1}, \ldots, b_{m}$ in $P$ such that $b_{k} \leqslant_{P} c_{k}$ and $f^{-1}(k)=\left[b_{k}, c_{k}\right]$ for each $k$.

In Section 2, we introduced the reduced interval system $\mathcal{I}^{\text {red }}(P)$ of a poset $P$. In Section 7, we will see that the reduced interval system is sometimes easier to handle than the full interval system.

Corollary 8. Let $P$ be a rooted poset. Then $\mathcal{I}(P)$ is partitionable if and only if $\mathcal{I}^{\text {red }}(P)$ is partitionable.

\section{Some concepts from topological combinatorics}

After having discussed basic properties of set systems and homomorphisms, we want to associate a cell complex to any given pair of set systems. Before doing that, we need to introduce some concepts from topological combinatorics. We also discuss some topological methods that will prove useful in later sections. We refer to Björner [3] for more information on the topic. 
In this paper, all cell complexes are combinatorially defined. A common situation is that each cell is indexed by a pair of sets $(X, Y)$, and the cells in the boundary of the cell are indexed by pairs $\left(X_{0}, Y_{0}\right) \neq(X, Y)$ satisfying $X_{0} \subseteq X$ and $Y_{0} \subseteq Y$. For convenience, we identify a cell complex with the family of combinatorial objects indexing its cells. It will always be clear from context how the cells are glued together.

For the purposes of this paper, a cell complex always has a finite number of cells, and the boundary of each cell is a union of lower-dimensional cells. We refer to an $i$ dimensional cell as an $i$-cell. We will find it convenient to include a virtual $(-1)$-cell in each cell complex. This is mainly because we will consider augmented cellular chain complexes and reduced homology, which corresponds to adding such a cell. Most of our cell complexes are regular, meaning that all attaching maps are homeomorphisms. The rare exceptions are quotients of regular complexes by subcomplexes.

We will use various techniques to establish homotopy equivalences between different cell complexes. The following useful result is known as the Contractible Subcomplex Lemma; see Hatcher [9, Prop. 0.17].

Lemma 9. Let $\Gamma$ be a cell complex, and let $\Gamma_{0}$ be a contractible subcomplex. Then $\Gamma \simeq$ $\Gamma / \Gamma_{0}$.

Recall that one may define the suspension $S \Gamma=S_{x, y} \Gamma$ of $\Gamma$ as the quotient of $\Gamma \times[-1,1]$ by collapsing $\Gamma \times\{-1\}$ to one point $x$ and $\Gamma \times\{1\}$ to another point $y$. Interpreting $[-1,1]$ as a cell complex with 0 -cells at $-1,0$, and 1 joined by the 1 -cells $(-1,0)$ and $(0,1)$, we may view the suspension as a cell complex. Indeed, if $\Gamma$ is regular, then so is $S \Gamma$. We identify $\Gamma$ with the subcomplex $\Gamma \times\{0\}$ of $S \Gamma$.

The cone $C \Gamma=C_{x} \Gamma$ of $\Gamma$ is the quotient of $\Gamma \times[0,1]$ by collapsing $\Gamma \times\{1\}$ to a point $x$; this is a subcomplex of $S \Gamma$. For a proof of the following fact, see Jonsson [10, Lemma $3.18]$.

Lemma 10. Let $\Gamma$ be a contractible cell complex, and let $\Gamma_{0}$ be a subcomplex. Then $\Gamma / \Gamma_{0} \simeq S \Gamma_{0}$.

Forman's discrete Morse theory [8] is a useful technique for establishing homotopy equivalences between cell complexes. In this paper, we restrict our attention to the special case of collapses in regular cell complexes.

Let $\mathcal{F}$ be a family of cells in a regular cell complex $\Gamma$, and assume that we may partition $\mathcal{F}$ into pairs of cells, each of the form $(\sigma, \tau)$, where $\sigma$ is a face of $\tau$ of codimension one. Suppose that we may order the pairs as $\left(\sigma_{1}, \tau_{1}\right), \ldots,\left(\sigma_{r}, \tau_{r}\right)$ such that $\sigma_{i}$ is not contained in $\tau_{j}$ unless $i \leqslant j$. Then we refer to the partition as a perfect acyclic matching on $\mathcal{F}$ and an acyclic matching on $\Gamma$. Almost by definition, we have the following result.

Lemma 11. Let $\Gamma_{0}$ be a subcomplex of a regular cell complex $\Gamma$, and assume that there exists a perfect acyclic matching on $\Gamma \backslash \Gamma_{0}$. Then $\Gamma$ admits a collapse to $\Gamma_{0}$. In particular, $\Gamma \simeq \Gamma_{0}$

We say that $\Gamma$ is collapsible if $\Gamma$ admits a collapse to a simplex (and hence to a single point). Collapsible complexes are contractible. 
As already alluded to, a common situation in this paper is that the nonempty cells of the complex are indexed by pairs $(X, Y)$ of sets. Suppose that we are given a matching in which each pair is of the form $(X \backslash\{x\}, Y),(X \cup\{x\}, Y)$, where $x$ is a fixed element. Then the matching is acyclic; we leave the proof to the reader.

Given two posets $P=\left(V(P), \leqslant_{P}\right)$ and $Q=\left(V(Q), \leqslant_{Q}\right)$, a poset map $f: P \rightarrow Q$ is a map $f: V(P) \rightarrow V(Q)$ such that $f(x) \leqslant_{Q} f(y)$ whenever $x \leqslant_{P} y$.

The face poset $P(\Gamma)$ of a cell complex $\Gamma$ is the poset consisting of all nonempty cells of $\Gamma$ ordered by inclusion. The order complex $\Delta(P)$ of a poset $P$ is the simplicial complex consisting of all chains of $P$. If $\Gamma$ is a regular cell complex, then $\Delta(P(\Gamma))$ is the barycentric subdivision of $\Gamma$, and the two complexes are homeomorphic.

Let $P$ be a poset. Given $x \in V(P)$, we let $P^{\leqslant x}$ denote the set of all $z \in V(P)$ such that $z \leqslant x$. The following result is known as Quillen's Fiber Lemma [16].

Lemma 12. Let $f: P \rightarrow Q$ be a poset map such that the fiber $\Delta\left(f^{-1}\left(Q^{\leqslant y}\right)\right)$ is contractible for each $y \in V(Q)$. Then $f$ induces a homotopy equivalence from $\Delta(P)$ to $\Delta(Q)$.

In this paper, the typical situation is that $P=P(\Gamma)$ for some regular cell complex $\Gamma$. In this case, $f^{-1}\left(Q^{\leqslant y}\right)$ coincides with the face poset $P\left(\Gamma_{y}\right)$ of some subcomplex $\Gamma_{y}$ of $\Gamma$, and $\Delta\left(f^{-1}\left(Q^{\leqslant y}\right)\right)=\Delta\left(P\left(\Gamma_{y}\right)\right) \cong \Gamma_{y}$.

Corollary 13. Let $\Gamma$ be a regular cell complex, and let $f: P(\Gamma) \rightarrow Q$ be a poset map such that $\Gamma_{y}$ is contractible for each $y \in V(Q)$, where $P\left(\Gamma_{y}\right)=f^{-1}\left(Q^{\leqslant y}\right)$. Then $f$ induces a homotopy equivalence from $\Gamma$ to $\Delta(Q)$. In particular, if $Q$ is the face poset of the regular cell complex $\Sigma$, then $f$ induces a homotopy equivalence from $\Gamma$ to $\Sigma$.

\section{$5 \quad$ Hom complexes of general set systems}

As already mentioned, Lovász introduced a cell complex $\operatorname{Hom}(G, H)$ with one vertex for each graph homomorphism from $G$ to $H$. In the language of the present paper, the vertices are indexed by homomorphisms between the independence systems of $G$ and $H$. Engström [6] generalized the construction to finite monotone systems, and the construction turns out to be straightforward to extend to any pair of finite set systems.

From now on, we only consider finite set systems. Let $\mathcal{S}$ and $\mathcal{T}$ be two such systems. We define $\operatorname{Hom}(\mathcal{S}, \mathcal{T})$ to be a cell complex in which each nonempty cell is indexed by a set function $F: V(\mathcal{S}) \rightarrow 2^{V(\mathcal{T})} \backslash\{\emptyset\}$ with the following property.

- We have that $f \in \operatorname{Hom}_{0}(\mathcal{S}, \mathcal{T})$ for every map $f: V(\mathcal{S}) \rightarrow V(\mathcal{T})$ such that $f(x) \in$ $F(x)$ for each $x \in V(\mathcal{S})$.

We write $f \in F$ if $f(x) \in F(x)$ for each $x \in V(\mathcal{S})$. By convention, the empty (-1)dimensional cell $\emptyset$ is always part of $\operatorname{Hom}(\mathcal{S}, \mathcal{T})$.

Assuming $V(\mathcal{S})=\left\{v_{1}, \ldots, v_{m}\right\}$, the closure of the cell indexed by $F$ is a product $\sigma_{1} \times \cdots \times \sigma_{m}$, where $\sigma_{i}$ is an $\left(\left|F\left(v_{i}\right)\right|-1\right)$-simplex. The faces of codimension one of the cell indexed by $F$ are those cells that are indexed by set functions obtained by removing 
one element from one of the sets $F\left(v_{i}\right)$. Note that we do not obtain a cell when removing an element from a set with only one element. The dimension of a cell is given by $\sum_{x \in V(\mathcal{S})}|F(x)|-|V(\mathcal{S})|$. See Babson and Kozlov [2] for more information about Hom complexes in the particular case of independence systems of graphs.

By Lemma 1, we may define a category Systems in which objects are set systems and morphisms are set system homomorphisms. Let Top be the category of topological spaces, in which morphisms are continuous maps. By the following result, we have a covariant functor $\operatorname{Hom}(\mathcal{S},-)$ from $\operatorname{Systems}$ to Top for each set system $\mathcal{S}$.

Proposition 14. For any set systems $\mathcal{S}, \mathcal{T}, \mathcal{U}$ and any homomorphism $h \in \operatorname{Hom}_{0}(\mathcal{T}, \mathcal{U})$, we obtain a cellular map

$$
h_{*}: \operatorname{Hom}(\mathcal{S}, \mathcal{T}) \rightarrow \operatorname{Hom}(\mathcal{S}, \mathcal{U})
$$

by mapping the cell in $\operatorname{Hom}(\mathcal{S}, \mathcal{T})$ indexed by $F$ to the cell in $\operatorname{Hom}(\mathcal{S}, \mathcal{U})$ indexed by $h F$.

Proof. Suppose that $g$ is a map from $\mathcal{S}$ to $\mathcal{U}$ such that $g \in h F$. This means that $g(x)=$ $h\left(\alpha_{x}\right)$ for some $\alpha_{x} \in F(x)$ for each $x \in V(\mathcal{S})$. By definition, we have that $f \in F$, where $f$ is the map defined by $f(x)=\alpha_{x}$. As a consequence, $f \in \operatorname{Hom}_{0}(\mathcal{S}, \mathcal{T})$, which yields that $g=h f \in \operatorname{Hom}_{0}(\mathcal{S}, \mathcal{U})$.

We will be interested in the reduced cellular homology of $\operatorname{Hom}(\mathcal{S}, \mathcal{T})$ with coefficients in $\mathbb{Z}$. For this, we need to fix notation and agree on some conventions regarding the associated chain groups and boundary maps.

For $j \geqslant 0$, we let $C_{j}[\mathcal{S} \rightarrow \mathcal{T}]$ denote the cellular chain group of degree $j$ of $\operatorname{Hom}(\mathcal{S}, \mathcal{T})$ with coefficients in $\mathbb{Z}$. This is a $\mathbb{Z}$-module with one generator, an oriented $j$-cell, for each $j$-cell of $\operatorname{Hom}(\mathcal{S}, \mathcal{T})$. A given cell $F$ is a direct product of simplices, and the corresponding oriented cell is the tensor product of the associated oriented simplices. For example, if $V(\mathcal{S})=\{1,2,3\}$ and $(F(1), F(2), F(3))=(\{a, b, c\},\{d\},\{e, f\})$, then the corresponding oriented cell is $a \wedge b \wedge c \otimes d \otimes e \wedge f$. We use the shorthand notation $\sigma_{1}\left|\sigma_{2}\right| \cdots \mid \sigma_{k}$ to denote the oriented cell $\sigma_{1} \otimes \sigma_{2} \otimes \cdots \otimes \sigma_{k}$.

The boundary map $\partial$ is defined on a generator $\sigma_{1}\left|\sigma_{2}\right| \cdots \mid \sigma_{k}$ by the recursive rule

$$
\partial\left(\sigma_{1}\left|\sigma_{2}\right| \cdots \mid \sigma_{k}\right)=\partial\left(\sigma_{1}\right)\left|\sigma_{2}\right| \cdots\left|\sigma_{k}+(-1)^{j+1} \sigma_{1}\right| \partial\left(\sigma_{2}|\cdots| \sigma_{k}\right),
$$

where $j$ is the degree of $\sigma_{1}$. The boundary of an oriented simplex $a_{0} a_{1} a_{2} \cdots a_{j}=a_{0} \wedge$ $a_{1} \wedge a_{2} \wedge \cdots \wedge a_{j}$ is given recursively by the usual rule $\partial\left(a_{0} a_{1} a_{2} \cdots a_{j}\right)=a_{1} a_{2} \cdots a_{j}-$ $a_{0} \partial\left(a_{1} a_{2} \cdots a_{j}\right)$. By convention, this is zero if $j=0$. For example,

$$
\partial(a b c|d e| f)=b c|d e| f-a c|d e| f+a b|d e| f-a b c|e| f+a b c|d| f .
$$

We will find it convenient to work with the augmented chain complex obtained by defining the chain group of degree -1 to be an infinite cyclic group generated by an element $e_{\emptyset}$ corresponding to the empty cell. We redefine $\partial$ on vertices, letting $\partial\left(a_{1}\left|a_{2}\right| \cdots \mid a_{k}\right)=e_{\emptyset}$. Let $\tilde{C}_{j}[\mathcal{S} \rightarrow \mathcal{T}]$ denote the augmented chain group of degree $j$; this group coincides with $C_{j}[\mathcal{S} \rightarrow \mathcal{T}]$ for $j \geqslant 0$. Let $\tilde{H}_{j}[\mathcal{S} \rightarrow \mathcal{T}]$ denote the associated reduced homology group.

We now state and prove one of the main results of the paper. The result relates the topology of certain Hom complexes to the partitionability of a set system. Let $\pi_{i}[\mathcal{S} \rightarrow \mathcal{T}]$ denote the $i$ th homotopy group of $\operatorname{Hom}(\mathcal{S}, \mathcal{T})$. 
Theorem 15. Let $\mathcal{S}$ be a partitionable set system. For any $\varphi \in \operatorname{Hom}_{0}\left(\mathcal{D}_{\omega(\mathcal{S})}, \mathcal{S}\right)$ and any set system $\mathcal{X}$, we have that the following hold.

(a) $\varphi$ induces a monomorphism from $\pi_{*}\left[\mathcal{X} \rightarrow \mathcal{D}_{\omega(\mathcal{S})}\right]$ to $\pi_{*}[\mathcal{X} \rightarrow \mathcal{S}]$. In particular, if $\operatorname{Hom}(\mathcal{X}, \mathcal{S})$ is $k$-connected for a given $k$, then so is $\operatorname{Hom}\left(\mathcal{X}, \mathcal{D}_{\omega(\mathcal{S})}\right)$.

(b) $\varphi$ induces a monomorphism from $\tilde{H}_{*}\left[\mathcal{X} \rightarrow \mathcal{D}_{\omega(\mathcal{S})}\right]$ to $\tilde{H}_{*}[\mathcal{X} \rightarrow \mathcal{S}]$.

Proof. Write $\omega=\omega(\mathcal{S})$. By definition of partitionability, there exists a homomorphism $h \in \operatorname{Hom}_{0}\left(\mathcal{S}, \mathcal{D}_{\omega}\right)$. We have that $h \varphi$ is bijective, because $h \varphi$ is a homomorphism from $\mathcal{D}_{\omega}$ to itself. By symmetry, we may assume that $h \varphi$ is the identity.

By Proposition $14, h$ induces a cellular map $h_{*}: \operatorname{Hom}(\mathcal{X}, \mathcal{S}) \rightarrow \operatorname{Hom}\left(\mathcal{X}, \mathcal{D}_{\omega}\right)$. Proposition 14 also yields that $\varphi$ induces a cellular map $\varphi_{*}: \operatorname{Hom}\left(\mathcal{X}, \mathcal{D}_{\omega}\right) \rightarrow \operatorname{Hom}(\mathcal{X}, \mathcal{S})$. Since $h \varphi$ is the identity map on $\mathcal{D}_{\omega}$, we obtain that $h_{*} \varphi_{*}$ is the identity map on $\operatorname{Hom}\left(\mathcal{X}, \mathcal{D}_{\omega}\right)$. We conclude that $\varphi_{*}$ induces monomorphisms as stated in the theorem.

For clarity, let us express part (b) of the theorem in the most practically useful form.

Corollary 16. Let $\mathcal{S}$ be a set system, and let $\varphi \in \operatorname{Hom}_{0}\left(\mathcal{D}_{\omega(\mathcal{S})}, \mathcal{S}\right)$. Suppose that there exists a set system $\mathcal{X}$ such that the map from $\tilde{H}_{*}\left[\mathcal{X} \rightarrow \mathcal{D}_{\omega(\mathcal{S})}\right]$ to $\tilde{H}_{*}[\mathcal{X} \rightarrow \mathcal{S}]$ induced by $\varphi$ is not a monomorphism. Then $\mathcal{S}$ is not partitionable.

One benefit of considering reduced homology is that Corollary 16 then covers the case that $\operatorname{Hom}_{0}\left(\mathcal{X}, \mathcal{D}_{\omega(\mathcal{S})}\right)$ is empty and $\operatorname{Hom}_{0}(\mathcal{X}, \mathcal{S})$ is nonempty. In this case, $\mathcal{S}$ is not partitionable by Lemma 1.

Let us say that $\mathcal{X}$ is a witness for a non-partitionable set system $\mathcal{S}$ if there is a homomorphism $\varphi \in \operatorname{Hom}_{0}\left(\mathcal{D}_{\omega(\mathcal{S})}, \mathcal{S}\right)$ such that the induced homomorphism in homology given in Corollary 16 fails to be a monomorphism. Note that $\omega(\mathcal{X}) \leqslant \omega(\mathcal{S})$, because otherwise $\operatorname{Hom}_{0}\left(\mathcal{X}, \mathcal{D}_{\omega(\mathcal{S})}\right)$ and $\operatorname{Hom}_{0}(\mathcal{X}, \mathcal{S})$ are both empty.

For the special case of rooted posets, the following result is immediate from the fact that the identity map on $V(P)$ defines a homomorphism from the interval system $\mathcal{I}(P)$ to the reduced interval system $\mathcal{I}^{\text {red }}(P)$.

Proposition 17. Let $\mathcal{X}$ be a set system, and let $P$ be a (finite) rooted poset. If $\mathcal{X}$ is a witness for $\mathcal{I}(P)$, then $\mathcal{X}$ is also a witness for $\mathcal{I}^{\text {red }}(P)$.

Remark 18. The converse is not always true. For example, assuming that $P$ is nonpartitionable, we have that $\mathcal{I}^{\text {red }}(P)$ is a witness for itself; the identity is a homomorphism from $\mathcal{I}^{\text {red }}(P)$ to itself, whereas there is no homomorphism from $\mathcal{I}^{\text {red }}(P)$ to $\mathcal{D}_{\omega(P)}$. Yet, $\mathcal{I}^{\text {red }}(P)$ is not a witness for $\mathcal{I}(P)$, because there is no homomorphism from $\mathcal{I}^{\text {red }}(P)$ to $\mathcal{I}(P)$. Namely, given such a homomorphism $\varphi$, the nonempty members of the family $\left\{\varphi^{-1}(\{x\}): x \in V(P)\right\}$ would constitute a partition of $\mathcal{I}^{\text {red }}(P)$ into $\omega(P)$ stable sets; there are as many nonempty members as there are coatoms in $P$. Such a partition does not exist. 
While $\operatorname{Hom}(\mathcal{S},-)$ defines a covariant functor for each $\mathcal{S}$, the dual construction does not yield a contravariant functor in general. For example, let

$$
\begin{aligned}
& \mathcal{S}=(\{a, b\},\{\emptyset,\{a, b\}\}), \\
& \mathcal{T}=(\{c\},\{\emptyset,\{c\}\}) \cong \mathcal{D}_{1}, \\
& \mathcal{U}=(\{d, e\},\{\emptyset,\{d\},\{e\}\}) \cong \mathcal{D}_{2} .
\end{aligned}
$$

Define $f:\{a, b\} \rightarrow\{c\}$ and $H:\{c\} \rightarrow 2^{\{d, e\}} \backslash\{\emptyset\}$ by $f(a)=f(b)=c$ and $H(c)=$ $\{d, e\}$. Then $H$ indexes a cell in $\operatorname{Hom}(\mathcal{T}, \mathcal{U})$, and $f \in \operatorname{Hom}_{0}(\mathcal{S}, \mathcal{T})$. However, $\operatorname{Hf}(a)=$ $H f(b)=\{d, e\}$, which does not index a cell in $\operatorname{Hom}(\mathcal{S}, \mathcal{U})$. For example, consider the map $g:\{a, b\} \rightarrow\{d, e\}$ given by $g(a)=d$ and $g(b)=e$. This map belongs to $H f$, but $g^{-1}(\{d\})=\{a\}$, which is not stable in $\mathcal{S}$, whereas $\{d\}$ is stable in $\mathcal{U}$.

As we will see in Section 6.2, we do get a contravariant functor $\operatorname{Hom}(-, \mathcal{U})$ if we restrict to the subcategory MonSystems of monotone systems.

\section{Hom complexes on monotone systems}

We consider Hom complexes $\operatorname{Hom}(\mathcal{S}, \mathcal{T})$ in the case that $\mathcal{S}$ is a monotone system, starting with the case $\mathcal{S}=\mathcal{D}_{m}$ in Section 6.1 and proceeding with general monotone systems in Section 6.2 .

\subsection{Hom complexes on discrete systems}

We give an overview of some basic properties of Hom complexes involving discrete systems $\mathcal{D}_{m}$. Before proceeding, let us recall that $\mathcal{D}_{m}$ is the independence system of the complete loopless graph $K_{m}$. By the work of Lovász [13], $K_{2}$ is a useful "test graph" for establishing lower bounds on the chromatic number of a graph. Babson and Kozlov [2] have obtained analogous results for general complete graphs.

For any set system $\mathcal{S}$, we may equip $\operatorname{Hom}\left(\mathcal{D}_{2}, \mathcal{S}\right)$ with the $\mathbb{Z}_{2}$-action given by $(X, Y) \rightarrow$ $(Y, X)$. This action is free if and only if each $v \in V(\mathcal{S})$ is contained in some stable set of $\mathcal{S}$. Recall from Section 3.1 that we refer to $\mathcal{S}$ itself as free if this is the case.

Proposition 19. For $m \leqslant n$, we have that $\operatorname{Hom}\left(\mathcal{D}_{m}, \mathcal{D}_{n}\right)$ is homotopy equivalent to a wedge of $(n-m)$-dimensional spheres. In fact, $\operatorname{Hom}\left(\mathcal{D}_{2}, \mathcal{D}_{n}\right)$ is $\mathbb{Z}_{2}$-homeomorphic to the $(n-2)$-sphere equipped with the antipodal action.

See Babson and Kozlov [2] for a proof. Čukić and Kozlov [5] and Engström [7] proved generalizations of the first statement. See Schultz [17, Example 4.5] for an alternative proof of the second statement.

For a graph $G$, the neighborhood complex $N(G)$ is the simplicial complex on the vertex set of $G$ in which a set $\sigma$ forms a face if and only if some vertex in $G$ is adjacent to all members of $\sigma$. The neighborhood complex was instrumental in Lovász's proof of Kneser's conjecture [13]. Identifying the independence system of $G$ with the independence complex 
$\operatorname{Ind}(G)$, it is well-known $[4,11]$ that $\operatorname{Hom}\left(\operatorname{Ind}\left(K_{2}\right), \operatorname{Ind}(G)\right)$ is homotopy equivalent to $N(G)$. Let us extend this result to general set systems.

For a set system $\mathcal{S}$, say that two elements $x, y \in V(\mathcal{S})$ are separated if no stable set of $\mathcal{S}$ contains both $x$ and $y$. Let $N(\mathcal{S})$ be the simplicial complex of all sets $\sigma \subseteq V(\mathcal{S})$ such that there is an element $x \in V(\mathcal{S})$ that is separated from all elements in $\sigma$. If $\mathcal{S}$ is the independence system of a graph $G$, then two elements are separated if and only if they are joined by an edge, and $N(\mathcal{S})$ coincides with $N(G)$.

For a given set system $\mathcal{S}$, it is convenient to identify a cell $F \in \operatorname{Hom}\left(\mathcal{D}_{m}, \mathcal{S}\right)$ with the corresponding $m$-tuple $(F(1), \ldots, F(m))$.

Proposition 20. For any set system $\mathcal{S}$, we have that $\operatorname{Hom}\left(\mathcal{D}_{2}, \mathcal{S}\right)$ is homotopy equivalent to $N(\mathcal{S})$.

Proof. Write $\Sigma=\operatorname{Hom}\left(\mathcal{D}_{2}, \mathcal{S}\right)$ and $Q=P(N(\mathcal{S}))$. A surjective poset map $\varphi: P(\Sigma) \rightarrow Q$ is given by mapping $(X, Y)$ to $X$. Namely, the map is well-defined, because any element in $X$ must be separated from any element in $Y$, which is nonempty. Moreover, the map is surjective, because if $X$ is a nonempty face of $N(\mathcal{S})$, then there is an element $y \in V(\mathcal{S})$ separated from $X$, which means that $(X,\{y\}) \in \Sigma$.

By Corollary 13, it suffices to prove the following.

- For each $X \in N(\mathcal{S}) \backslash\{\emptyset\}$, we have that $\Sigma_{X}$ is contractible, where $\Sigma_{X}$ is the subcomplex of $\Sigma$ with face poset

$$
\varphi^{-1}\left(Q^{\subseteq X}\right)=\varphi^{-1}\left(2^{X} \backslash\{\emptyset\}\right) .
$$

Let $y \in V(\mathcal{S})$ be any element such that $(X,\{y\}) \in \Sigma_{X}$. Then $\left(X_{0}, Y \cup\{y\}\right) \in \Sigma_{X}$ whenever $\left(X_{0}, Y\right) \in \Sigma_{X}$. Using Lemma 11, we may collapse $\Sigma_{X}$ to the subcomplex $\Sigma_{X}^{\prime}$ consisting of all cells of the form $\left(X_{0},\{y\}\right)$ such that $X_{0} \subseteq X$. Specifically, a perfect acyclic matching on $\Sigma_{X} \backslash \Sigma_{X}^{\prime}$ is given by pairing $\left(X_{0}, Y \backslash\{y\}\right)$ with $\left(X_{0}, Y \cup\{y\}\right)$ for each $\left(X_{0}, Y\right) \in \Sigma_{X}$ such that $X_{0} \subseteq X$ and $Y \backslash\{y\} \neq \emptyset$. Since $\Sigma_{X}^{\prime}$ is an $(|X|-1)$-simplex, $\Sigma_{X}$ is collapsible.

Corollary 21. If $\mathcal{S}$ is partitionable and $\chi(\mathcal{S})=n$, then $\tilde{H}_{n-2}(N(\mathcal{S}) ; \mathbb{Z})$ is infinite.

Proof. This follows from Theorem 15 and Propositions 19 and 20.

The next result extends a famous theorem of Lovász [13] to general set systems.

Proposition 22. Let $\mathcal{S}$ be a set system. If $N(\mathcal{S})$ is $(n-2)$-connected, then $\chi(\mathcal{S})>n$.

Proof. If $\mathcal{S}$ is not free, then $\chi(\mathcal{S})=\infty$. Assume that $\mathcal{S}$ is free; each $v \in V(\mathcal{S})$ is contained in some stable set of $\mathcal{S}$. By Proposition 20,N(S) is homotopy equivalent to $\operatorname{Hom}\left(\mathcal{D}_{2}, \mathcal{S}\right)$. Suppose that there exists a map $f \in \operatorname{Hom}_{0}\left(\mathcal{S}, \mathcal{D}_{n}\right)$. Then there is a map $f_{*}: \operatorname{Hom}\left(\mathcal{D}_{2}, \mathcal{S}\right) \rightarrow \operatorname{Hom}\left(\mathcal{D}_{2}, \mathcal{D}_{n}\right) \cong_{\mathbb{Z}_{2}} S^{n-2}$, and this map commutes with the $\mathbb{Z}_{2}$-action. Yet, since $\operatorname{Hom}\left(\mathcal{D}_{2}, \mathcal{S}\right)$ is a free $(n-2)$-connected $\mathbb{Z}_{2}$-complex, there is a $\mathbb{Z}_{2}$-map from $S^{n-1}$ to $\operatorname{Hom}\left(\mathcal{D}_{2}, \mathcal{S}\right)$; see Matoušek [14, Prop. 5.3.2 (iv)]. As a consequence, we have a $\mathbb{Z}_{2}$-map from $S^{n-1}$ to $S^{n-2}$, which contradicts the Borsuk-Ulam Theorem. 


\subsection{Hom complexes on general monotone systems}

Given the discussion in Section 6.1, it seems reasonable to ask whether the cell complex $\operatorname{Hom}\left(\mathcal{D}_{2}, \mathcal{S}\right) \simeq \mathcal{N}(\mathcal{S})$ tells us something useful about the partitionability of the set system $\mathcal{S}$. Disappointingly, this does not seem to be the case for many interesting set systems $\mathcal{S}$, including interval systems of rooted posets. Indeed, we will see that any monotone system fails to be a witness for interval systems. On the positive side, we show that the category MonSystems of monotone systems has nice functorial properties.

Let $\mathcal{S}$ be a set system, and write $\omega=\omega(\mathcal{S})$. We say that $\mathcal{S}$ admits an optimal cover if there are $\omega$ stable sets $\sigma_{1}, \ldots, \sigma_{\omega}$ such that

$$
V(\mathcal{S})=\bigcup_{j=1}^{\omega} \sigma_{j} .
$$

Given a copartition $\left(c_{1}, \ldots, c_{m}\right)$, we have that each $\sigma_{j}$ contains at most one $c_{i}$. In particular, if $m=\omega$, then each $\sigma_{j}$ contains exactly one $c_{i}$, and each $c_{i}$ belongs to exactly one $\sigma_{j}$.

Note that any partitionable set system admits an optimal cover, but the converse is not true in general. Let us consider two important special cases.

- Let $\mathcal{S}$ be a monotone system. Then $\mathcal{S}$ admits an optimal cover if and only if $\mathcal{S}$ is partitionable. Namely, given an optimal cover $\left(\sigma_{1}, \ldots, \sigma_{\omega(\mathcal{S})}\right)$, we obtain a partition by removing elements from the sets $\sigma_{j}$ until each element in the ground set appears in exactly one set $\sigma_{j}$.

- Suppose that $P$ is any rooted poset with minimal element 0 . Then $\mathcal{I}(P)$ admits an optimal cover. Namely, $V(P)$ is the union over all coatoms $c$ of the intervals $[0, c]$. By Proposition 6, the number of coatoms is $\omega(P)$.

To conclude, the situation for monotone systems is completely different from that for set systems of rooted posets.

By the following result, monotone systems are not witnesses for systems admitting an optimal cover.

Proposition 23. Let $\mathcal{X}$ be a monotone system, and let $\mathcal{S}$ be a set system admitting an optimal cover. Then any map $\varphi \in \operatorname{Hom}_{0}\left(\mathcal{D}_{\omega(\mathcal{S})}, \mathcal{S}\right)$ induces monomorphisms from $\pi_{*}\left[\mathcal{X} \rightarrow \mathcal{D}_{\omega(\mathcal{S})}\right]$ to $\pi_{*}[\mathcal{X} \rightarrow \mathcal{S}]$ and from $\tilde{H}_{*}\left[\mathcal{X} \rightarrow \mathcal{D}_{\omega(\mathcal{S})}\right]$ to $\tilde{H}_{*}[\mathcal{X} \rightarrow \mathcal{S}]$.

Proof. If $\operatorname{Hom}_{0}(\mathcal{X}, \mathcal{S})$ is empty, then so is $\operatorname{Hom}_{0}\left(\mathcal{X}, \mathcal{D}_{\omega(\mathcal{S})}\right)$; hence we may assume that $\operatorname{Hom}_{0}(\mathcal{X}, \mathcal{S})$ is nonempty.

Write $\omega=\omega(\mathcal{S})$. Let $\left(\sigma_{1}, \ldots, \sigma_{\omega}\right)$ be an optimal cover of $\mathcal{S}$. Define a map $h: V(\mathcal{S}) \rightarrow$ $\{1, \ldots, \omega\}$ in the following manner. For each $v \in V(\mathcal{S})$, pick any element $k \in\{1, \ldots, \omega\}$ such that $v \in \sigma_{k}$, and define $h(v)=k$. While $h$ is typically not a homomorphism from $\mathcal{S}$ to $\mathcal{D}_{\omega}$, we claim that $h$ still induces a cellular map from $\operatorname{Hom}(\mathcal{X}, \mathcal{S})$ to $\operatorname{Hom}\left(\mathcal{X}, \mathcal{D}_{\omega}\right)$.

To prove the claim, consider a cell in $\operatorname{Hom}(\mathcal{X}, \mathcal{S})$ indexed by the set function $F$. We need to show that every $g \in h F$ is a homomorphism from $\mathcal{X}$ to $\mathcal{D}_{\omega}$, which is equivalent 
to saying that the preimage under $g$ of every element in $\{1, \ldots, \omega\}$ is stable in $\mathcal{X}$. Now, write $A_{k}=g^{-1}(\{k\})$. For each $a \in A_{k}$, we have that $k=h\left(b_{a}\right)$ for some $b_{a} \in F(a)$. By construction, all elements in the set $B_{k}=\left\{b_{a}: a \in A_{k}\right\}$ belong to $\sigma_{k}$. Let $f \in F$ be such that $f(a)=b_{a}$ for $a \in A_{k}$. Since $\sigma_{k}$ is stable in $\mathcal{S}$ and $f \in \operatorname{Hom}_{0}(\mathcal{X}, \mathcal{S})$, we have that $f^{-1}\left(\sigma_{k}\right)$ is stable in $\mathcal{X}$. Yet,

$$
f^{-1}\left(\sigma_{k}\right) \supseteq f^{-1}\left(B_{k}\right) \supseteq A_{k}
$$

Since $\mathcal{X}$ is monotone, we deduce that $A_{k}$ is stable.

To conclude, $h$ induces a cellular map $h_{*}: \operatorname{Hom}(\mathcal{X}, \mathcal{S}) \rightarrow \operatorname{Hom}\left(\mathcal{X}, \mathcal{D}_{\omega}\right)$. We also have that $\varphi$ induces a cellular map $\varphi_{*}: \operatorname{Hom}\left(\mathcal{X}, \mathcal{D}_{\omega}\right) \rightarrow \operatorname{Hom}(\mathcal{X}, \mathcal{S})$. Now, $h \varphi$ is a bijection, because the restriction of $h$ to the set of elements of any $\omega$-copartition is necessarily a bijection. As a consequence, the composition $h_{*} \varphi_{*}$ must be an isomorphism, which yields the proposition.

The following result generalizes the fact that a monotone system $\mathcal{S}$ is partitionable whenever $\mathcal{S}$ admits an optimal cover.

Proposition 24. Let $\mathcal{X}$ be a monotone system, and let $\mathcal{S}$ be a set system admitting an optimal cover. If $\operatorname{Hom}_{0}(\mathcal{X}, \mathcal{S})$ is nonempty, then $\chi(\mathcal{X}) \leqslant \omega(\mathcal{S})$.

Proof. Let $f \in \operatorname{Hom}_{0}(\mathcal{X}, \mathcal{S})$. Given an optimal cover $\left(\sigma_{1}, \ldots, \sigma_{\omega(\mathcal{S})}\right)$ of $\mathcal{S}$, we have that $V(\mathcal{X})$ is the union of the stable sets $\rho_{i}=f^{-1}\left(\sigma_{i}\right)$. Removing elements from the sets $\rho_{i}$ until each element appears in exactly one set, we obtain a partition of $\mathcal{X}$ into $\omega(\mathcal{S})$ stable sets.

In Section 5, we observed that $\operatorname{Hom}(-, \mathcal{U})$ does not define a contravariant functor from Systems to Top. By the following result, the situation becomes much nicer if we restrict to the subcategory MonSystems of monotone systems.

Proposition 25. Let $\mathcal{S}$ be any monotone system, let $\mathcal{T}$ and $\mathcal{U}$ be any set systems, and let $f \in \operatorname{Hom}_{0}(\mathcal{S}, \mathcal{T})$. Then we obtain a cellular map

$$
f^{*}: \operatorname{Hom}(\mathcal{T}, \mathcal{U}) \rightarrow \operatorname{Hom}(\mathcal{S}, \mathcal{U})
$$

by mapping the cell in $\operatorname{Hom}(\mathcal{T}, \mathcal{U})$ indexed by $H$ to the cell in $\operatorname{Hom}(\mathcal{S}, \mathcal{U})$ indexed by $H f$.

Proof. Suppose that $g$ is a map from $\mathcal{S}$ to $\mathcal{U}$ such that $g \in H f$. To prove the proposition, it suffices to show that $\delta=g^{-1}(\tau)$ is a stable set in $\mathcal{S}$ for each stable set $\tau$ in $\mathcal{U}$.

Let $\left\{y_{1}, \ldots, y_{k}\right\}$ be the image of $f$, and define $\sigma_{j}=f^{-1}\left(y_{j}\right)$ for $1 \leqslant j \leqslant k$. Note that the ground set $X$ of $\mathcal{S}$ is the disjoint union of the sets $\sigma_{1}, \ldots, \sigma_{k}$. Let $Y$ and $Z$ be the ground sets of $\mathcal{T}$ and $\mathcal{U}$, respectively, and define a map $h: Y \rightarrow Z$ via the following procedure. For each $j$ such that $\sigma_{j} \cap \delta \neq \emptyset$, let $x_{j} \in \sigma_{j} \cap \delta$. If $\sigma_{j} \cap \delta=\emptyset$, pick an arbitrary $x_{j} \in \sigma_{j}$. For $1 \leqslant j \leqslant k$, define

$$
h\left(y_{j}\right)=g\left(x_{j}\right) \in H f\left(x_{j}\right)=H\left(y_{j}\right) .
$$


For $y \in Y \backslash\left\{y_{1}, \ldots, y_{k}\right\}$, define $h(y)$ to be an arbitrary element in $H(y)$. It is clear that $h \in H$ and hence that $h \in \operatorname{Hom}_{0}(\mathcal{T}, \mathcal{U})$.

Let $\tilde{g}=h f \in \operatorname{Hom}_{0}(\mathcal{S}, \mathcal{U})$. For each $x \in \sigma_{j}$, we have that $\tilde{g}(x)=h f(x)=h f\left(x_{j}\right)=$ $g\left(x_{j}\right)$. In particular, $\tilde{g}(x) \in \tau$ if and only if $\delta \cap \sigma_{j} \neq \emptyset$. We conclude that

$$
\tilde{g}^{-1}(\tau)=\bigcup_{\delta \cap \sigma_{j} \neq \emptyset} \sigma_{j} \supseteq \delta .
$$

Now, $\tilde{g}$ is a homomorphism, which yields that $\tilde{g}^{-1}(\tau)$ is stable in $\mathcal{S}$. Since $\mathcal{S}$ is monotone, we deduce that the subset $\delta$ is also stable in $\mathcal{S}$.

\section{Hom complexes on some non-monotone systems}

By Proposition 23, only non-monotone set systems can be witnesses for set systems admitting an optimal cover. This section gives a few examples of non-monotone witnesses for some reduced interval systems of simplicial complexes. The purpose is merely to illustrate Theorem 15; the simplicial complexes under consideration are easily seen to be non-partitionable by direct examination.

In Section 7.1, we provide a detailed analysis of the interval system $\mathcal{I}\left(L_{3}\right)$ of the 3chain $L_{3}$, which is the poset on the ground set $\{1,2,3\}$ with the total order $1<2<3$. This interval system is non-monotone; the stable sets are all subsets of $\{1,2,3\}$ except $\{1,3\}$. Besides showing that $\mathcal{I}\left(L_{3}\right)$ is a witness for certain reduced interval systems, we compute the homotopy type of $\operatorname{Hom}\left(\mathcal{I}\left(L_{3}\right), \mathcal{D}_{n}\right)$, which turns out to be that of a wedge of $(n-1)$-dimensional spheres.

In Section 7.2, we consider a few other non-monotone set systems and briefly discuss their potential as witnesses.

\subsection{Hom complexes on the 3-chain}

Note that a map $f:\{1,2,3\} \rightarrow V(\mathcal{S})$ defines a homomorphism from $\mathcal{I}\left(L_{3}\right)$ to the set system $\mathcal{S}$ if and only if every stable set of $\mathcal{S}$ containing $f(1)$ and $f(3)$ also contains $f(2)$. We may identify each nonempty cell of $\operatorname{Hom}\left(\mathcal{I}\left(L_{3}\right), \mathcal{S}\right)$ with a triple $\left(X_{1}, X_{2}, X_{3}\right)$, where $X_{1}, X_{2}, X_{3}$ are nonempty subsets of $V(\mathcal{S})$ such that each choice of elements $a_{i} \in X_{i}$ defines a homomorphism $i \mapsto a_{i}$ from $\mathcal{I}\left(L_{3}\right)$ to $\mathcal{S}$. Equivalently, for every stable set $\sigma$ of $\mathcal{S}$, if there are elements $a_{1} \in X_{1}$ and $a_{3} \in X_{3}$ such that $a_{1}, a_{3} \in \sigma$, then $X_{2} \subseteq \sigma$.

For a given set system $\mathcal{S}$, define $\Gamma(\mathcal{S})$ to be the family of all pairs $\left(X_{1}, X_{3}\right)$ such that $\left(X_{1}, X_{2}, X_{3}\right) \in \operatorname{Hom}\left(\mathcal{I}\left(L_{3}\right), \mathcal{S}\right)$ for some $X_{2}$. The members of $\Gamma(\mathcal{S})$ index the nonempty cells of a cell complex. This cell complex is a subcomplex of the product of two $(|V(\mathcal{S})|-1)$ simplices; the cell indexed by a given pair $\left(X_{1}, X_{3}\right)$ is the product of an $\left(\left|X_{1}\right|-1\right)$-simplex and an $\left(\left|X_{3}\right|-1\right)$-simplex. We identify $\Gamma(\mathcal{S})$ with the corresponding cell complex.

A surjective cell complex map $\varphi: \operatorname{Hom}\left(\mathcal{I}\left(L_{3}\right), \mathcal{S}\right) \rightarrow \Gamma(\mathcal{S})$ is given by

$$
\varphi\left(X_{1}, X_{2}, X_{3}\right)=\left(X_{1}, X_{3}\right)
$$


Proposition 26. The map $\varphi$ induces a homotopy equivalence between the complexes $\operatorname{Hom}\left(\mathcal{I}\left(L_{3}\right), \mathcal{S}\right)$ and $\Gamma(\mathcal{S})$.

Proof. Write $\Sigma=\operatorname{Hom}\left(\mathcal{I}\left(L_{3}\right), \mathcal{S}\right)$ and $\Gamma=\Gamma(\mathcal{S})$. We may view $\varphi$ as a poset map from $P(\Sigma)$ to $P(\Gamma)$. Corollary 13 yields that it suffices to prove the following.

- For every $\gamma \in \Gamma \backslash\{\emptyset\}$, we have that $\Sigma_{\gamma}$ is contractible, where

$$
\Sigma_{\gamma}=\varphi^{-1}(\{\rho \in \Gamma: \rho \leqslant \gamma\}) .
$$

Consider $\gamma=\left(X_{1}, X_{3}\right)$. Let $\mu \in V$ be any element such that $\left(X_{1},\{\mu\}, X_{3}\right) \in \Sigma_{\gamma}$. Then $\left(Y_{1}, Y_{2} \cup\{\mu\}, Y_{3}\right) \in \Sigma_{\gamma}$ whenever $\left(Y_{1}, Y_{2}, Y_{3}\right) \in \Sigma_{\gamma}$. Using Lemma 11, we may collapse $\Sigma_{\gamma}$ to the subcomplex $\Sigma_{\gamma}^{\prime}$ consisting of all cells of the form $\left(Y_{1},\{\mu\}, Y_{3}\right)$ such that $Y_{1} \subseteq X_{1}$ and $Y_{3} \subseteq X_{3}$. Specifically, a perfect acyclic matching on $\Sigma_{\gamma} \backslash \Sigma_{\gamma}^{\prime}$ is given by pairing $\left(Y_{1}, Y_{2} \backslash\{\mu\}, Y_{3}\right)$ with $\left(Y_{1}, Y_{2} \cup\{\mu\}, Y_{3}\right)$ for each $\left(Y_{1}, Y_{2}, Y_{3}\right) \in \Sigma_{\gamma}$ such that $Y_{2} \backslash\{\mu\} \neq \emptyset$. Since $\Sigma_{\gamma}^{\prime}$ is the product of an $\left(\left|X_{1}\right|-1\right)$-simplex and an $\left(\left|X_{3}\right|-1\right)$-simplex, we deduce that $\Sigma_{\gamma}^{\prime}$ is collapsible. As a consequence, the same is true for $\Sigma_{\gamma}$.

From now on, we consider $\Gamma(\mathcal{S})$ instead of $\operatorname{Hom}\left(\mathcal{I}\left(L_{3}\right), \mathcal{S}\right)$. Let $\Gamma_{0}\left(\mathcal{D}_{n}\right)$ be the subcomplex of $\Gamma\left(\mathcal{D}_{n}\right)$ consisting of all pairs $(X, Y)$ such that $X \cap Y=\emptyset$. By Proposition 19, we have that

$$
\Gamma_{0}(\mathcal{D})=\operatorname{Hom}\left(\mathcal{D}_{2}, \mathcal{D}_{n}\right) \cong S^{n-2} .
$$

Proposition 27. We have that

$$
\Gamma\left(\mathcal{D}_{n}\right) \simeq \bigvee_{n-1} S^{n-1}
$$

Hence

$$
\tilde{H}_{i}\left(\Gamma\left(\mathcal{D}_{n}\right) ; \mathbb{Z}\right) \cong \begin{cases}\mathbb{Z}^{n-1} & \text { if } i=n-1, \\ 0 & \text { if } i \neq n-1 .\end{cases}
$$

Proof. Note that $\Gamma\left(\mathcal{D}_{n}\right)$ consists of all pairs $(X, Y)$ such that $|X \cap Y| \leqslant 1$. For $i \in$ $\{1, \ldots, n\}$, let $\Gamma_{i}$ be the subcomplex of $\Gamma\left(\mathcal{D}_{n}\right)$ consisting of all pairs $(X, Y)$ such that $X \cap Y \subseteq\{n\}$.

We have that $\Gamma_{i}$ is collapsible. Namely, let $\Sigma_{i}$ be the subcomplex consisting of all cells $(A,\{i\})$ such that $A$ is a nonempty subset of $\{1, \ldots, n\}$. Using Lemma 11, we obtain a collapse from $\Gamma_{i}$ to $\Sigma_{i}$ by pairing $(A, B \backslash\{i\})$ with $(A, B \cup\{i\})$ whenever $B \backslash\{i\} \neq \emptyset$. Since $\Sigma_{i}$ is an $(n-1)$-simplex, we obtain the desired result.

Observing that $\Gamma_{i} \cap \Gamma_{j}=\Gamma_{0}=\Gamma_{0}\left(\mathcal{D}_{n}\right)$ whenever $i \neq j$, we conclude that

$$
\Gamma \simeq \Gamma / \Gamma_{n} \cong \bigvee_{i=1}^{n-1} \Gamma_{i} / \Gamma_{0} \simeq \bigvee_{n-1} S \Gamma_{0} \cong \bigvee_{n-1} S^{n-1}
$$

the homotopy equivalences are consequences of Lemmas 9 and 10 . 


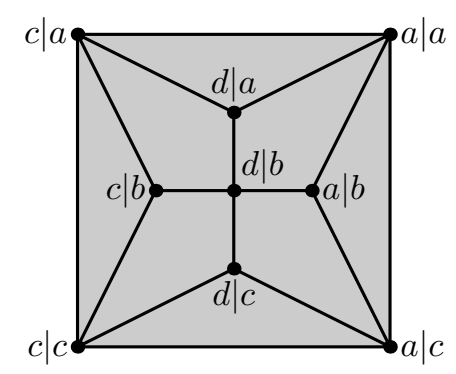

Figure 1: The cycle $a|a c+a c| a-c|a c-a c| c$ is a boundary in the chain complex of $\Gamma\left(\mathcal{I}^{\text {red }}\left(K_{2}^{2}\right)\right)$.

As promised, we now show that the 3 -chain is a witness for certain reduced interval systems of simplicial complexes. First we consider the simplicial complex $K_{2}^{2}$ on the vertex set $\{a, b, c, d\}$ with maximal faces $\{a, b\}$ and $\{c, d\}$.

Proposition 28. $\mathcal{I}\left(L_{3}\right)$ is a witness for $\mathcal{I}^{\mathrm{red}}\left(K_{2}^{2}\right)$.

Proof. As described in Section 5, we let $x_{1} \cdots x_{k} \mid y_{1} \cdots y_{\ell}$ denote the oriented cell corresponding to a given cell $\left(\left\{x_{1}, \ldots, x_{k}\right\},\left\{y_{1}, \ldots, y_{\ell}\right\}\right)$ in $\Gamma(\mathcal{S})$. We have that $\Gamma\left(\mathcal{D}_{2}\right)$ is the boundary of a square, and the fundamental cycle is

$$
z=1|12+12| 1-2|12-12| 2 ;
$$

see Section 5 for the definition of the boundary operator $\partial$. Let $f \in \operatorname{Hom}_{0}\left(\mathcal{D}_{2}, \mathcal{I}^{\text {red }}\left(K_{2}^{2}\right)\right)$ be defined by $f(1)=a$ and $f(2)=c$. Note that $f$ maps $z$ to the cycle

$$
z^{\prime}=a|a c+a c| a-c|a c-a c| c
$$

in the chain complex of $\Gamma\left(\mathcal{I}^{\text {red }}\left(K_{2}^{2}\right)\right)$. Yet, $z^{\prime}$ is the boundary of the element

$$
c^{\prime}=a|a b c-a d| b c-a c d|c+c d| b c-c|a b c+c d| a b+a c d|a-a d| a b .
$$

See Figure 1 for a geometric illustration. As a consequence, $f$ does not induce a monomorphism in homology; hence $\mathcal{I}\left(L_{3}\right)$ is a witness for $\mathcal{I}^{\text {red }}\left(K_{2}^{2}\right)$.

Remark 29. Using Pilarczyk's computer program homchain [15], one can verify that

$$
\tilde{H}_{i}\left(\Gamma\left(\mathcal{I}^{\text {red }}\left(K_{2}^{2}\right)\right) ; \mathbb{Z}\right) \cong \begin{cases}\mathbb{Z}^{2} & \text { if } i=2, \\ 0 & \text { otherwise. }\end{cases}
$$

It is possible to extend Proposition 28 to a larger class of systems. Let $K_{1}^{n-2}+K_{2}^{2}$ be the simplicial complex on the vertex set $\left\{x_{1}, \ldots, x_{n-2}, a, b, c, d\right\}$ with maximal faces $\left\{x_{1}\right\}, \ldots,\left\{x_{n-2}\right\},\{a, b\}$ and $\{c, d\}$.

Proposition 30. $\mathcal{I}\left(L_{3}\right)$ is a witness for $\mathcal{T}=\mathcal{I}^{\text {red }}\left(K_{1}^{n-2}+K_{2}^{2}\right)$. 
Proof. For an oriented cell $A \mid B$ and an element $x$ not in $A$ or $B$, define

$$
A|B \triangleright x=A| B x-(-1)^{\delta} x A \mid B,
$$

where $\delta$ is the degree of the cell $A|B ; \delta=| A|+| B \mid-2$. For $|A|+|B|>2$, note that

$$
\partial(A \mid B \triangleright x)=\partial(A \mid B) \triangleright x+ \begin{cases}0 & \text { if }|A| \geqslant 2,|B| \geqslant 2 \\ (-1)^{\delta} x \mid B & \text { if }|A|=1,|B| \geqslant 2 \\ -(-1)^{\delta} A \mid x & \text { if }|A| \geqslant 2,|B|=1\end{cases}
$$

Extending $\triangleright$ linearly, we obtain, for $z^{\prime}$ and $c^{\prime}$ defined as in (4) and (5), that

$$
\partial\left(c^{\prime} \triangleright x\right)=\partial\left(c^{\prime}\right) \triangleright x=z^{\prime} \triangleright x,
$$

because all terms of the forms $x \mid B$ and $A \mid x$ cancel out. Recursively defining $c \triangleright x_{1} \cdots x_{k}=$ $\left(c \triangleright x_{1} \cdots x_{k-1}\right) \triangleright x_{k}$, a simple induction argument yields that

$$
\partial\left(c^{\prime} \triangleright x_{1} \cdots x_{n-2}\right)=\partial\left(c^{\prime}\right) \triangleright x_{1} \cdots x_{n-2}=z^{\prime} \triangleright x_{1} \cdots x_{n-2} .
$$

In particular, $z^{\prime} \triangleright x_{1} \cdots x_{n-2}$ is both a cycle and a boundary in the chain complex of $\Gamma(\mathcal{T})$.

Now, define a homomorphism $f$ from $\mathcal{D}_{n}$ to $\mathcal{T}$ by $f(i)=x_{i}$ for $i \leqslant n-2, f(n-1)=a$ and $f(n)=c$. Letting $z$ be defined as in (3), the cycle $z^{\prime} \triangleright x_{1} \cdots x_{n-2}$ is the image under $f$ of $w=z \triangleright 12 \cdots(n-2)$, which is a cycle of degree $n-1$ in the chain complex of the cell complex $\Gamma\left(\mathcal{D}_{n}\right)$. Since the dimension of the latter complex is $n-1$, the cycle $w$ is not a boundary. In particular, the homology map induced by $f$ is not a monomorphism.

Corollary 31. Let $\mathcal{U}$ be a set system such that $\omega(\mathcal{U})=n$ and such that there is a homomorphism from $\mathcal{I}^{\text {red }}\left(K_{1}^{n-2}+K_{2}^{2}\right)$ to $\mathcal{U}$. Then $\mathcal{I}\left(L_{3}\right)$ is a witness for $\mathcal{U}$. In particular, $\mathcal{I}\left(L_{3}\right)$ is a witness for the reduced interval system of any simplicial complex containing a link isomorphic to $K_{2}^{2}$.

For the latter statement, a homomorphism is given by mapping $K_{2}^{2}$ bijectively to the link under consideration and mapping the isolated vertices in $K_{1}^{n-2}$ bijectively to the maximal faces outside the link.

Remark 32. Regrettably, $\mathcal{I}\left(L_{3}\right)$ fails to be a witness for the reduced and unreduced interval systems of any pure simplicial complex with the property that every link of dimension at least one is connected. In particular, $\mathcal{I}\left(L_{3}\right)$ cannot be a witness for the interval system of a Cohen-Macaulay complex. We omit the proof, which is both lengthy and technical. Recall from Section 1 that it is not known whether there exist any non-partitionable Cohen-Macaulay complexes.

\subsection{Other potential witnesses}

In this section, we provide two examples of other set systems that might be potentially useful as witnesses. Indeed, it turns out that both systems are witnesses for the reduced interval system of $K_{2}^{2}$. The proofs are even simpler than that of Proposition 28, which 
settled the analogous fact for the reduced interval system of the 3-chain. It remains an open question whether the counterparts of the more general Proposition 30 are true.

The first example is the interval system of the poset $X$ consisting of the five elements $x_{1}, x_{2}, y, z_{1}, z_{2}$ satisfying $x_{i} \leqslant y$ and $y \leqslant z_{i}$ for all $i \in\{1,2\}$; the only incomparable pairs of elements are $\left(x_{1}, x_{2}\right)$ and $\left(z_{1}, z_{2}\right)$. One may check that we obtain a homomorphism $f$ from $\mathcal{I}(X)$ to $\mathcal{I}^{\text {red }}\left(K_{2}^{2}\right)$ by defining

$$
f\left(x_{1}\right)=a, f\left(x_{2}\right)=c, f(y)=\emptyset, f\left(z_{1}\right)=b, f\left(z_{2}\right)=d .
$$

In particular, $\operatorname{Hom}\left(\mathcal{I}(X), \mathcal{I}^{\text {red }}\left(K_{2}^{2}\right)\right)$ is not the empty complex, which implies that there is no nonzero reduced homology in degree -1 . Yet, it is easy to see that $X$ is not partitionable, which is equivalent to saying that $\operatorname{Hom}\left(\mathcal{I}(X), \mathcal{D}_{2}\right)$ is the empty complex. We conclude that this complex has nonvanishing reduced homology in degree -1 . As a consequence, $\mathcal{I}(X)$ is a witness for the reduced interval system of $K_{2}^{2}$.

The second example is the set system $\mathcal{F}$ on the ground set $\{1,2,3\}$ with stable sets

$$
\emptyset,\{1\},\{2\},\{3\},\{1,2\},\{1,2,3\} .
$$

Equivalently, $\{1,3\}$ and $\{2,3\}$ are the non-stable sets. Given another set system $\mathcal{T}$, we may identify a cell $F \in \operatorname{Hom}(\mathcal{F}, \mathcal{T}$ ) (and the associated oriented cell) with the triple $F(1)|F(2)| F(3)$. One may note that $\operatorname{Hom}\left(\mathcal{F}, \mathcal{D}_{2}\right)$ is the disjoint union of two edges, one with endpoints $1|1| 1$ and $1|1| 2$ and one with endpoints $2|2| 1$ and $2|2| 2$. In particular, there is nonvanishing homology in degree 0 , and the homology is generated by the homology class of the cycle

$$
z=1|1| 1-2|2| 2 .
$$

Let $f \in \operatorname{Hom}_{0}\left(\mathcal{D}_{2}, \mathcal{I}^{\text {red }}\left(K_{2}^{2}\right)\right)$ be defined by $f(1)=a$ and $f(2)=c$. We have that $f$ induces a map from $\operatorname{Hom}\left(\mathcal{F}, \mathcal{D}_{2}\right)$ to $\operatorname{Hom}\left(\mathcal{F}, \mathcal{I}^{\text {red }}\left(K_{2}^{2}\right)\right)$, and the associated chain map sends $z$ to the cycle

$$
z^{\prime}=a|a| a-c|c| c
$$

Now, $z^{\prime}$ is the boundary of

$$
c|c| c d-c|c \emptyset| d+c \emptyset|\emptyset| d+\emptyset|\emptyset| d b-a \emptyset|\emptyset| b+a|a \emptyset| b-a|a| a b .
$$

In particular, the map in homology induced by $f$ is not a monomorphism, which means that $\mathcal{F}$ is a witness for $\Lambda$.

\section{Categorical properties of set systems}

Recall that Systems is the category in which objects are set systems and morphisms are set system homomorphisms. In this section, we discuss properties of this category. To start with, we observe that any void system of the form $(\{x\}, \emptyset)$ is a terminal object. There is no initial object, but we could introduce one by adding the system $(\emptyset,\{\emptyset\})$ and introducing an "empty" homomorphism from this system to any other system. For the purposes of the present section, we will not need any initial object. 
For two families $\Delta_{1}$ and $\Delta_{2}$ of subsets of $V$ and $W$, respectively, define

$$
\Delta_{1} * \Delta_{2}=\left\{\delta_{1} \uplus \delta_{2}: \delta_{1} \in \Delta_{1}, \delta_{2} \in \Delta_{2}\right\} \subseteq 2^{V \uplus W} .
$$

Proposition 33. The categorical coproduct of two set systems $\mathcal{S}_{1}$ and $\mathcal{S}_{2}$ is the set system

$$
\mathcal{S}_{1} * \mathcal{S}_{2}=\left(V\left(\mathcal{S}_{1}\right) \uplus V\left(\mathcal{S}_{2}\right), \Delta\left(\mathcal{S}_{1}\right) * \Delta\left(\mathcal{S}_{2}\right)\right) .
$$

Proof. Consider the inclusion map $\iota_{i}: V\left(\mathcal{S}_{i}\right) \rightarrow V\left(\mathcal{S}_{1}\right) \uplus V\left(\mathcal{S}_{2}\right)$ for $i \in\{1,2\}$. The preimage under $\iota_{i}$ of a stable set $\sigma_{1} \uplus \sigma_{2} \in \Delta\left(\mathcal{S}_{1}\right) * \Delta\left(\mathcal{S}_{2}\right)$ is $\sigma_{i}$, which is stable in $\mathcal{S}_{i}$; hence $\iota_{i} \in \operatorname{Hom}_{0}\left(\mathcal{S}_{i}, \mathcal{S}_{1} * \mathcal{S}_{2}\right)$ for $i \in\{1,2\}$.

Next, let $\mathcal{T}$ be any set system. Given morphisms $f_{i} \in \operatorname{Hom}_{0}\left(\mathcal{S}_{i}, \mathcal{T}\right)$, define $f: V\left(\mathcal{S}_{1}\right) \uplus$ $V\left(\mathcal{S}_{2}\right) \rightarrow V(\mathcal{T})$ by $f(v)=f_{1}(v)$ if $v \in V\left(\mathcal{S}_{1}\right)$ and $f(v)=f_{2}(v)$ if $v \in V\left(\mathcal{S}_{2}\right)$. This is the unique morphism in $\operatorname{Hom}_{0}\left(\mathcal{S}_{1} * \mathcal{S}_{2}, \mathcal{T}\right)$ satisfying $f \iota_{i}=f_{i}$ for $i \in\{1,2\}$. Namely, uniqueness is clear, and given any stable set $\sigma$ of $\mathcal{T}$, the preimage of $\sigma$ is $f^{-1}(\sigma)=f_{1}^{-1}(\sigma) \uplus f_{2}^{-1}(\sigma)$, hence stable.

We have that a map $f: \mathcal{S} * \mathcal{T} \rightarrow \mathcal{U}$ is a homomorphism if and only if the restriction of $f$ to each of $\mathcal{S}$ and $\mathcal{T}$ is a homomorphism. As a consequence, we have the following result.

Proposition 34. For any set systems $\mathcal{S}, \mathcal{T}$, and $\mathcal{U}$, we have that the cell complexes $\operatorname{Hom}(\mathcal{S} * \mathcal{T}, \mathcal{U})$ and $\operatorname{Hom}(\mathcal{S}, \mathcal{U}) \times \operatorname{Hom}(\mathcal{T}, \mathcal{U})$ are isomorphic.

The shape of $\operatorname{Hom}(\mathcal{S}, \mathcal{T} * \mathcal{U})$ depends on the involved set systems; we confine ourselves to a special case. The separability graph of a set system $\mathcal{S}$ is the graph on the vertex set $V(\mathcal{S})$ in which two vertices $u$ and $v$ are joined by an edge if and only if $u$ and $v$ are separated in $\mathcal{S}$ (see Section 6.1).

Proposition 35. Assume that the separability graph of $\mathcal{S}$ is connected and that $|V(\mathcal{S})| \geqslant$ 2. Moreover, assume that $\mathcal{T}$ and $\mathcal{U}$ are both free (see Section 3.1). Then $\operatorname{Hom}(\mathcal{S}, \mathcal{T} * \mathcal{U})$ is isomorphic to the disjoint union of $\operatorname{Hom}(\mathcal{S}, \mathcal{T})$ and $\operatorname{Hom}(\mathcal{S}, \mathcal{U})$.

Proof. Since $V(\mathcal{S})$ has size at least two, it suffices to show that the image of each $f \in$ $\operatorname{Hom}_{0}(\mathcal{S}, \mathcal{T} * \mathcal{U})$ is contained in either $V(\mathcal{T})$ or $V(\mathcal{U})$. Assume the opposite. Since the separability graph of $\mathcal{S}$ is connected, there are two separated elements $x$ and $y$ such that $f(x) \in V(\mathcal{T})$ and $f(y) \in V(\mathcal{U})$. Since $\mathcal{T}$ and $\mathcal{U}$ are free, there are stable sets $\delta_{1} \in \Delta(\mathcal{T})$ and $\delta_{2} \in \Delta(\mathcal{U})$ such that $f(x) \in \delta_{1}$ and $f(y) \in \delta_{2}$. Yet, $f^{-1}\left(\delta_{1} \uplus \delta_{2}\right)$ contains both $x$ and $y$ and is hence not stable, a contradiction.

See Babson and Kozlov [2, §2.4] for the restrictions of Propositions 34 and 35 to independence systems of graphs.

Proposition 36. Let $\mathcal{S}_{1}$ and $\mathcal{S}_{2}$ be set systems such that the empty set is stable in both systems. Then

$$
\left\{\begin{array}{l}
\omega\left(\mathcal{S}_{1} * \mathcal{S}_{2}\right)=\max \left\{\omega\left(\mathcal{S}_{1}\right), \omega\left(\mathcal{S}_{2}\right)\right\} \\
\chi\left(\mathcal{S}_{1} * \mathcal{S}_{2}\right)=\max \left\{\chi\left(\mathcal{S}_{1}\right), \chi\left(\mathcal{S}_{2}\right)\right\}
\end{array}\right.
$$


Proof. By the existence of a homomorphism in $\operatorname{Hom}_{0}\left(\mathcal{S}_{i}, \mathcal{S}_{1} * \mathcal{S}_{2}\right)$, any homomorphism in $\operatorname{Hom}_{0}\left(\mathcal{S}_{1} * \mathcal{S}_{2}, \mathcal{D}_{n}\right)$ extends to a homomorphism in $\operatorname{Hom}_{0}\left(\mathcal{S}_{i}, \mathcal{D}_{n}\right)$ for $i=1$, 2 . Thus $\chi\left(\mathcal{S}_{1} * \mathcal{S}_{2}\right) \geqslant \max \left\{\chi\left(\mathcal{S}_{1}\right), \chi\left(\mathcal{S}_{2}\right)\right\}$.

Conversely, any two homomorphisms in $\operatorname{Hom}_{0}\left(\mathcal{S}_{1}, \mathcal{D}_{n}\right)$ and $\operatorname{Hom}_{0}\left(\mathcal{S}_{2}, \mathcal{D}_{n}\right)$ extend to a homomorphism in $\operatorname{Hom}_{0}\left(\mathcal{S}_{1} * \mathcal{S}_{2}, \mathcal{D}_{n}\right)$. Since the empty set belongs to $\Delta\left(\mathcal{S}_{i}\right)$, we have that $\operatorname{Hom}_{0}\left(\mathcal{S}_{i}, \mathcal{D}_{n}\right)$ is nonempty whenever $n \geqslant \chi\left(\mathcal{S}_{i}\right)$. Thus $\chi\left(\mathcal{S}_{1} * \mathcal{S}_{2}\right) \leqslant \max \left\{\chi\left(\mathcal{S}_{1}\right), \chi\left(\mathcal{S}_{2}\right)\right\}$.

By the existence of a homomorphism in $\operatorname{Hom}_{0}\left(\mathcal{S}_{i}, \mathcal{S}_{1} * \mathcal{S}_{2}\right)$, any homomorphism in $\operatorname{Hom}_{0}\left(\mathcal{D}_{m}, \mathcal{S}_{i}\right)$ extends to a homomorphism in $\operatorname{Hom}_{0}\left(\mathcal{D}_{m}, \mathcal{S}_{1} * \mathcal{S}_{2}\right)$ for $i=1,2$. Thus $\omega\left(\mathcal{S}_{1} * \mathcal{S}_{2}\right) \geqslant \max \left\{\omega\left(\mathcal{S}_{1}\right), \chi\left(\mathcal{S}_{2}\right)\right\}$.

Conversely, first note that $\mathcal{S}_{1} * \mathcal{S}_{2}$ is nonfree (see Section 3.1) whenever $\mathcal{S}_{1}$ or $\mathcal{S}_{2}$ is nonfree; hence $\omega\left(\mathcal{S}_{1} * \mathcal{S}_{2}\right)=\max \left\{\omega\left(\mathcal{S}_{1}\right), \chi\left(\mathcal{S}_{2}\right)\right\}=\infty$ by Proposition 4. Suppose that $\mathcal{S}_{1}$ and $\mathcal{S}_{2}$ are both free. Let $f \in \operatorname{Hom}_{0}\left(\mathcal{D}_{m}, \mathcal{S}_{1} * \mathcal{S}_{2}\right)$. Since both set systems are free, any two elements $y_{1} \in V\left(\mathcal{S}_{1}\right)$ and $y_{2} \in V\left(\mathcal{S}_{2}\right)$ appear in a common stable set in $\Delta_{1} * \Delta_{2}$. In particular, the image of $f$ must be a subset of either $V\left(\mathcal{S}_{1}\right)$ or $V\left(\mathcal{S}_{2}\right)$, meaning that we may view $f$ as a member of $\operatorname{Hom}_{0}\left(\mathcal{D}_{m}, \mathcal{S}_{i}\right)$ for either $i=1$ or $i=2$. Thus $\omega\left(\mathcal{S}_{1} * \mathcal{S}_{2}\right) \leqslant \max \left\{\omega\left(\mathcal{S}_{1}\right), \omega\left(\mathcal{S}_{2}\right)\right\}$.

Proposition 37. The categorical product of two set systems $\mathcal{T}_{1}$ and $\mathcal{T}_{2}$ is the set system

$$
\mathcal{T}_{1} \times \mathcal{T}_{2}=\left(V\left(\mathcal{T}_{1}\right) \times V\left(\mathcal{T}_{2}\right),\left\{\sigma_{1} \times V\left(\mathcal{T}_{2}\right): \sigma_{1} \in \Delta\left(\mathcal{T}_{1}\right)\right\} \cup\left\{V\left(\mathcal{T}_{1}\right) \times \sigma_{2}: \sigma_{2} \in \Delta\left(\mathcal{T}_{2}\right)\right\}\right) .
$$

Proof. Consider the projection map $\pi_{i}: V\left(\mathcal{T}_{1}\right) \times V\left(\mathcal{T}_{2}\right) \rightarrow V\left(\mathcal{T}_{i}\right)$ for $i \in\{1,2\}$. The preimage under $\pi_{1}$ of a stable set $\sigma_{1} \in \Delta\left(\mathcal{T}_{1}\right)$ is $\sigma_{1} \times V\left(\mathcal{T}_{2}\right)$, which is stable in $\mathcal{T}_{1} \times \mathcal{T}_{2}$. Similarly, the preimage under $\pi_{2}$ of every stable set is stable. In particular, $\pi_{i} \in \operatorname{Hom}_{0}\left(\mathcal{T}_{1} \times\right.$ $\left.\mathcal{T}_{2}, \mathcal{T}_{i}\right)$ for $i \in\{1,2\}$.

Next, let $\mathcal{S}$ be any set system. Given morphisms $f_{i} \in \operatorname{Hom}_{0}\left(\mathcal{S}, \mathcal{T}_{i}\right)$, define $f: V(\mathcal{S}) \rightarrow$ $V\left(\mathcal{T}_{1}\right) \times V\left(\mathcal{T}_{2}\right)$ by $f(v)=\left(f_{1}(v), f_{2}(v)\right)$. This is the unique morphism in $\operatorname{Hom}_{0}\left(\mathcal{S}, \mathcal{T}_{1} \times \mathcal{T}_{2}\right)$ satisfying $\pi_{i} f=f_{i}$ for $i \in\{1,2\}$. Namely, uniqueness is clear, and given any stable sets $\sigma_{1} \in \Delta\left(\mathcal{T}_{1}\right)$ and $\sigma_{2} \in \Delta\left(\mathcal{T}_{2}\right)$, the preimages of $\sigma_{1} \times V\left(\mathcal{T}_{2}\right)$ and $V\left(\mathcal{T}_{1}\right) \times \sigma_{2}$ are $f_{1}^{-1}\left(\sigma_{1}\right)$ and $f_{2}^{-1}\left(\sigma_{2}\right)$, respectively, hence stable.

Proposition 38. Let $\mathcal{T}_{1}$ and $\mathcal{T}_{2}$ be set systems. Then

$$
\left\{\begin{array}{l}
\omega\left(\mathcal{T}_{1} \times \mathcal{T}_{2}\right)=\min \left\{\omega\left(\mathcal{T}_{1}\right), \omega\left(\mathcal{T}_{2}\right)\right\} \\
\chi\left(\mathcal{T}_{1} \times \mathcal{T}_{2}\right)=\min \left\{\chi\left(\mathcal{T}_{1}\right), \chi\left(\mathcal{T}_{2}\right)\right\}
\end{array}\right.
$$

Proof. By the existence of a homomorphism in $\operatorname{Hom}_{0}\left(\mathcal{T}_{1} \times \mathcal{T}_{2}, \mathcal{T}_{i}\right)$, any homomorphism in $\operatorname{Hom}_{0}\left(\mathcal{D}_{m}, \mathcal{T}_{1} \times \mathcal{T}_{2}\right)$ extends to a homomorphism in $\operatorname{Hom}_{0}\left(\mathcal{D}_{m}, \mathcal{T}_{i}\right)$ for $i=1,2$. Thus $\omega\left(\mathcal{T}_{1} \times \mathcal{T}_{2}\right) \leqslant \min \left\{\omega\left(\mathcal{T}_{1}\right), \omega\left(\mathcal{T}_{2}\right)\right\}$

Conversely, any two homomorphisms in $\operatorname{Hom}_{0}\left(\mathcal{D}_{m}, \mathcal{T}_{1}\right)$ and $\operatorname{Hom}_{0}\left(\mathcal{D}_{m}, \mathcal{T}_{2}\right)$ extend to a homomorphism in $\operatorname{Hom}_{0}\left(\mathcal{D}_{m}, \mathcal{T}_{1} \times \mathcal{T}_{2}\right)$. As a consequence, $\omega\left(\mathcal{T}_{1} \times \mathcal{T}_{2}\right) \leqslant \min \left\{\omega\left(\mathcal{T}_{1}\right), \omega\left(\mathcal{T}_{2}\right)\right\}$.

By the existence of a homomorphism in $\operatorname{Hom}_{0}\left(\mathcal{T}_{1} \times \mathcal{T}_{2}, \mathcal{T}_{i}\right)$, any homomorphism in $\operatorname{Hom}_{0}\left(\mathcal{T}_{i}, \mathcal{D}_{n}\right)$ extends to a homomorphism in $\operatorname{Hom}_{0}\left(\mathcal{T}_{1} \times \mathcal{T}_{2}, \mathcal{D}_{n}\right)$ for $i=1,2$. Thus $\chi\left(\mathcal{T}_{1} \times\right.$ $\left.\mathcal{T}_{2}\right) \leqslant \min \left\{\chi\left(\mathcal{T}_{1}\right), \chi\left(\mathcal{T}_{2}\right)\right\}$

Conversely, let $f \in \operatorname{Hom}_{0}\left(\mathcal{T}_{1} \times \mathcal{T}_{2}, \mathcal{D}_{n}\right)$. Suppose that $k, \ell \in\{1, \ldots, n\}$ are elements such that $f^{-1}(k)=\sigma_{1} \times V\left(\mathcal{T}_{2}\right)$ and $f^{-1}(\ell)=V\left(\mathcal{T}_{1}\right) \times \sigma_{2}$ for some nonempty $\sigma_{1} \subseteq V\left(\mathcal{T}_{1}\right)$ 
and $\sigma_{2} \subseteq V\left(\mathcal{T}_{2}\right)$. Then $f^{-1}(k) \cap f^{-1}(\ell)=\sigma_{1} \times \sigma_{2} \neq \emptyset$, a contradiction. We conclude that the preimages $f^{-1}(k)$ are either all of the form $\sigma_{1} \times V\left(\mathcal{T}_{2}\right)$ or all of the form $V\left(\mathcal{T}_{1}\right) \times \sigma_{2}$. In the former case, we obtain a homomorphism $f_{1} \in \operatorname{Hom}_{0}\left(\mathcal{T}_{1}, \mathcal{D}_{n}\right)$ by defining $f_{1}(x)=$ $f(x, y)$, where we may pick $y$ arbitrarily. The latter case is treated analogously. Thus $\chi\left(\mathcal{T}_{1} \times \mathcal{T}_{2}\right) \geqslant \min \left\{\chi\left(\mathcal{T}_{1}\right), \chi\left(\mathcal{T}_{2}\right)\right\}$.

The categorical product is different if we restrict our attention to the subcategory MonSystems of monotone systems. More precisely, in this category, the product of two monotone systems $\mathcal{T}_{1}$ and $\mathcal{T}_{2}$ is the monotone system

$$
\left(V\left(\mathcal{T}_{1}\right) \times V\left(\mathcal{T}_{2}\right),\left\{E \subseteq V\left(\mathcal{T}_{1}\right) \times V\left(\mathcal{T}_{2}\right): \pi_{1}(E) \in \Delta\left(\mathcal{T}_{1}\right) \text { or } \pi_{2}(E) \in \Delta\left(\mathcal{T}_{2}\right)\right\}\right)
$$

Indeed, this is the monotone system on $V\left(\mathcal{T}_{1}\right) \times V\left(\mathcal{T}_{2}\right)$ in which the simplicial complex of stable sets is minimal with the property that all stable sets of $\mathcal{T}_{1} \times \mathcal{T}_{2}$ belong to the complex.

Acknowledgement. I thank an anonymous referee for several valuable comments.

\section{References}

[1] E. Babson and D. N. Kozlov, Proof of the Lovász conjecture, Ann. of Math. 165 (2007), 965-1007.

[2] E. Babson and D. N. Kozlov, Complexes of graph homomorphisms, Israel J. Math. 152 (2006), 285-312.

[3] A. Björner, Topological Methods, Handbook of Combinatorics, R. Graham, M. Grötschel and L. Lovász, (eds.), North-Holland, Amsterdam, 1995, 1819-1872.

[4] P. Csorba, On the simple $\mathbb{Z}_{2}$-homotopy types of graph complexes and their simple $\mathbb{Z}_{2}$-universality, Canad. Math. Bull. 51 (2008), 535-544.

[5] S. Lj. Čukić and D. N. Kozlov, Higher connectivity of graph coloring complexes. Int. Math. Res. Not. 2005 (2005), no. 25, 1543-1562.

[6] A. Engström, Cohomological Ramsey theory, Preprint, 2010. arXiv:1002.4074

[7] A. Engström, Set partition complexes, Discrete Comput. Geom. 40 (2008), 357-364.

[8] R. Forman, Morse theory for cell complexes, Adv. Math. 134 (1998), 90-145.

[9] A. Hatcher, Algebraic Topology, Cambridge University Press, 2002.

[10] J. Jonsson, Simplicial Complexes of Graphs, Lecture Notes in Mathematics 1928, Springer, 2008.

[11] D. N. Kozlov, Simple homotopy types of Hom-complexes, neighborhood complexes, Lovász complexes, and atom crosscut complexes, Topology Appl. 153 (2006), no. 14, 2445-2454.

[12] D. Kozlov, Combinatorial Algebraic Topology, Springer, 2007. 
[13] L. Lovász, Kneser's conjecture, chromatic number, and homotopy, J. Combinatorial Theory 25 (1978), 319-324.

[14] J. Matoušek, Using the Borsuk-Ulam Theorem, Springer, 2003.

[15] P. Pilarczyk, Computational Homology Program (CHomP), advanced version, 2004. Available from http://chomp.rutgers.edu/advanced/

[16] D. Quillen, Homotopy properties of the poset of nontrivial $p$-subgroups of a group, Adv. Math. 28 (1978), 101-128.

[17] C. Schultz, Graph colorings, spaces of edges and spaces of circuits, Adv. Math. 221 (2009), no. 6, 1733-1756.

[18] R. P. Stanley, Combinatorics and Commutative Algebra, Birkhäuser, 1983. 